\title{
Buchbesprechungen*
}

\section{Die Bestandteile der Lebensmittel und deren Bestimmung}

Beiträge zur schnellen Bestimmung wichtiger Radionuclide $\left({ }^{89} \mathrm{Sr},{ }^{90} \mathrm{Sr},{ }^{131} \mathrm{~J}\right.$ und ${ }^{137} \mathrm{Cs}$ ) in Lebensmitteln und Wasser. Dissertation von P. F. JAGERHUBER unter Leitung von K. G. Bergner. Technische Hochschule Stuttgart 1963.

Die Dissertation befaßt sich ausführlich mit den Problemen der Bestimmung biologisch bedeutsamer Radionuclide in Lebensmitteln und Wasser. Ausgehend von der Ủberlegung, daß wirkungsvolle Schutzmaßnahmen bei einer Erhöhung der Umweltradioaktivität nur ergriffen werden können, wenn die Meßergebnisse über die Konzentrationen der wichtigsten Radionuclide in Lebensmitteln und Wasser innerhalb kurzer Zeit nach der Probenahme vorliegen, werden Schnellmethoden zur Bestimmung dieser Radionuclide vorgeschlagen. Verf. gibt zunächst einen Úberblick über allgemeine Tatsachen und Probleme der Umweltradioaktivität, über die Möglichkeiten ihrer Utberwachung und über die Anforderungen, die nach seiner Meinung an die Untersuchung der Lebensmittel und des Wassers zu stellen sind. Nach grundsätzlichen Erörterungen über die Möglichkeiten der Messung von Radionucliden wird auf die verschiedenen Voraussetzungen für Schnellmethoden, wie Einflüsse der Probenmengen und der Meßzeit sowie die Vorbereitung des Probenmaterials eingegangen. Beim eigentlichen Gegenstand der Arbeit, der Entwicklung von Schnellmethoden, wird auf bereits bekannten Verfahren der Bestimmung aufgebaut. Das Ergebnis dieser Untersuchungen wird am Schluß der Arbeit in Form von Arbeitsvorschriften zusammengefaßt. Hier findet sich zunächst eine Vorschrift zur Bestimmung der Radionuclide ${ }^{131} \mathrm{~J}$, ${ }^{90} \mathrm{Sr},{ }^{89} \mathrm{Sr}$ und ${ }^{137} \mathrm{Cs}$ in Frischmilch. Die vier Radionuclide können danach in einer Probe bestimmt werden, wobei das Verfahren die Beobachtungen verschiedener Autoren kombiniert. Auf einer englischen Arbeit fußend wird ${ }^{131} \mathrm{~J}$ durch Gammamessung des Milcheiweißes, das aus der mit Formaldehyd vorbehandelten Milch durch Trichloressigsäurefällung gewonnen wurde, bestimmt. Aus dem Filtrat dieser Eiweißfällung werden durch Carbonatfällung und anschließende klassische Nitrattrennung die Sr-Isotope - ebenfalls in Anlehnung an die erwähnte englische Arbeit - abgetrennt. Auf Grund der Messung eines Sr-Carbonat- und eines Y-Oxalatpräparates, das aus dem Ungleichgewicht ausgefällt wurde, werden dieKonzentrationen an ${ }^{90} \mathrm{Sr}$ und ${ }^{89} \mathrm{Sr}$ berechnet. Die Zeitersparnis ergibt sich hierbei durch das Vermeiden der Veraschung und durch die Fällung des Y-Oxalatpräparates aus dem Ungleichgewicht. ${ }^{137} \mathrm{Cs}$ wird aus dem Filtrat der Carbonatfällung an einen Kationenaustauscher adsorbiert und nach Elution mit HCl und Fällung als Kieselwolframat gammaspektrometrisch gemessen. Neben der Einzelmessung der Gammastrahler wird auch die gammaspektrometrische Bestimmung der Gammastrahler nebeneinander unter Berücksichtigung ihrer gegenseitigen Beeinflussung besprochen. Bei der ${ }^{131} \mathrm{~J}$-Bestimmung in Wasser wird die Methode der trägerfreien Adsorption des Jods an einer Silberjodidsäule mit anschließender Gammamessung des Silberjodids gewählt. Für die Bestimmung von ${ }^{137} \mathrm{Cs}$, ${ }^{90} \mathrm{Sr}$ und ${ }^{89} \mathrm{Sr}$ in festen Lebensmitteln und Wasser wird eine eigene Arbeitsvorschrift angegeben. Nach Veraschung des Untersuchungsmaterials und Lösen der Asche mit HCl wird ${ }^{137} \mathrm{Cs}$ an eine Ammoniumphosphormolybdatsäule adsorbiert und anschließend wieder gammaspektrometrisch ausgewertet. Die Fällung der Sr-Isotope wird zusammen mit dem Ca als nichtisotopem Träger als Oxalate bei $\mathrm{pH} 2$ durchgeführt. Nach Überführung der Oxalate in die Nitrate und Zusatz von Sr-Träger wird wie bei Milch der übliche Trennungsgang ausgeführt.

Die Arbeit stellt eine wertvolle Sichtung und Utberprüfung bisher gebräuchlicher Methoden der Bestimmung von Radionucliden dar und kombiniert einige von ihnen in geschickter Weise. Ob das Ergebnis in jedem Fall als Schnellmethode bezeichnet werden kann, erscheint allerdings zweifelhaft.

W. Stadelmann (München)

\section{Die einzelnen Lebensmittel (Chemie, Technologie, Analytik)}

W. Ziesack: Unsere Nahrungsmittel. 340 S. mit 265 Abb. und 7 Taf. Stuttgart: Franckh'sche Verlagshandlung 1963. Gzl. 29.50 DM.

Das Buch richtet sich als Handbuch und Nachschlagewerk an Erzeuger, Händler und Verbraucher, es soll der Hauswirtschaftslehrerin wie dem Biologielehrer als nützliches Nachschlagewerk bei der Vorbereitung des Unterrichts dienen. Es fragt sich, ob das Buch diesem Zweck dienen kann. Bei der Beschreibung der einzelnen Lebensmittel wird eine strenge Gliederung

* Einschließlich Besprechungen von Diplomarbeiten und Dissertationen. 